\title{
Drying and Rehydration of Calcium Alginate Gels
}

\author{
Rob Vreeker • Liangbin Li • Yapeng Fang • \\ Ingrid Appelqvist • Eduardo Mendes
}

Received: 9 November 2007 / Accepted: 15 April 2008/Published online: 26 June 2008

(C) Springer Science + Business Media, LLC 2008

\begin{abstract}
In this paper, we study the rehydration properties of air-dried calcium alginate gel beads. Rehydration is shown to depend on alginate source (i.e. mannuronic to guluronic acid ratio) and the salt concentration in the rehydration medium. Rehydration curves are described adequately by the empirical Weibull equation. Wide-angle $\mathrm{X}$-ray diffraction measurements are performed to obtain information on the microstructure of dried alginate gels. The X-ray diffraction patterns provide evidence for forma-
\end{abstract}

R. Vreeker $(\bowtie)$

Unilever Food and Health Research Institute,

Olivier van Noortlaan 120 ,

3133 AT Vlaardingen, The Netherlands

e-mail: rob.vreeker@unilever.com

\section{L. $\mathrm{Li}$}

National Synchrotron Radiation Laboratory and Department of Polymer Science and Engineering, University of Science and Technology of China,

Anhui 230026, China

Y. Fang

Glyn O. Phillips Hydrocolloid Research Center,

North East Wales Institute,

Plas Coch, Mold Road,

Wrexham LL11 2AW, UK

\section{Appelqvist}

Commonwealth Scientific and Industrial Research Organisation,

Riverside Corporate Park, 11 Julius Avenue,

North Ryde, New South Wales 2113, Australia

E. Mendes

Section Nanostructured Materials,

Faculty of Chemical Engineering,

Delft University of Technology,

Julianalaan 136,

2628 BL Delft, The Netherlands tion of ordered domains in which alginate polymers are laterally associated. Formation of ordered structures during drying is found to have a large impact on rehydration properties. Lateral association of alginate chains is reduced (and rehydration improved) by removing excess calcium ions from the gel beads in a washing step prior to air drying. In addition, rehydration properties of mixed alginate-carboxymethyl cellulose (CMC) gel beads are investigated. The presence of CMC in the gel matrix is found to reduce lateral association of alginate chains during drying and to improve rehydration properties.

Keywords Alginate · Egg-box · Aggregation · Drying · Rehydration $\cdot$ Weibull

\section{Introduction}

Drying is a method of food preservation frequently used in the food industry. Unfortunately, drying and subsequent rehydration often results in a decline of food quality. This decrease is related to (irreversible) changes in the food microstructure during drying. Understanding the mechanism underlying these changes and their impact on the rehydration properties is essential for improving the quality of dried foods. From a physicochemical perspective, dried foods may be considered as a blend of biopolymeric materials. Thus, there is a requirement to understand and manipulate rehydration behaviour of biopolymer materials in water. The requirements with respect to rehydration are likely to depend on the type of food considered. In some cases, e.g. instant soups or sauces, it is desirable to have a rapid rehydration process in order to prevent product defects, such as grittiness caused by undissolved biopolymeric particles and to deliver the required texture in a short 
period of time. In other cases, it may be more advantageous to have very slow rehydration behaviour to prevent sogginess. Therefore, it is important to understand the key parameters that control the kinetics of rehydration. Knowledge about this topic is still somewhat limited, although various studies have appeared recently ${ }^{1-5}$.

In this paper, we study the rehydration properties of airdried calcium alginate gel beads. Alginate is used in (fabricated) food products because of its thickening or gelling properties and can be considered as a model system for more complex food materials. It is a family of linear copolymers of $(1 \rightarrow 4)$-linked $\beta$-D-mannuronic acid $(M)$ and $\alpha$-L guluronic acid $(\mathrm{G})$ residues. $\mathrm{G}$ and $\mathrm{M}$ residues are present in varying proportions and sequences depending on the alginic acid source ${ }^{6}$. Alginate gelation occurs when divalent cations (usually $\mathrm{Ca}^{++}$) interact with blocks of guluronic acid residues. This behaviour results in the formation of a three-dimensional gel network and is usually described by the "egg-box model"7. Two different methods are known for the preparation of alginate gels in the presence of $\mathrm{Ca}^{++}$, i.e. internal gelation and diffusion setting. The latter method was used in this study to prepare alginate gel beads. The beads were prepared using two commercial alginates with different guluronic to mannuronic acid ratios. In addition, "mixed" gel beads were prepared using a mixture of alginate and carboxymethyl cellulose (CMC). The beads were dried in air and subsequently rehydrated in aqueous salt solutions. The kinetics of rehydration was measured and analyzed using the empirical Weibull model ${ }^{8}$. Wide-angle X-ray diffraction measurements were performed to obtain information on the microstructure of dried alginate gel beads. The X-ray diffraction patterns provide evidence for formation of ordered domains. The nature of the domains was elucidated by comparing diffraction patterns of gel beads to those of alginate fibers (prepared from the same materials). The formation of ordered structures and their impact on the rehydration properties of alginate beads is discussed.

\section{Materials and Methods}

\section{Materials}

Two different alginates were used in this study. A highviscosity-grade sodium alginate derived from Macrocystis pyrifera was obtained from Sigma Aldrich Chemie. The alginate has a guluronic acid content of approximately $39 \%$ and will be referred to as "low-G alginate". A high gel strength sodium alginate (Manugel DMB) derived from Laminaria hyperborea was obtained from ISP Alginates. This alginate has a high guluronic acid content $(>55 \%)$ and will be referred to as "high-G alginate".
Preparation of Alginate Gel Beads

Sodium alginate was dissolved in Millipore water at a concentration of $1 \mathrm{wt} . \%$. Gel beads were prepared by dripping the sodium alginate solution from a syringe into $500 \mathrm{ml} 1$ wt. $\% \mathrm{CaCl}_{2} \cdot 2 \mathrm{H}_{2} \mathrm{O}$ solution under constant stirring. Beads were kept in the calcium chloride solution for $1 \mathrm{~h}$ and then taken out. The diameter of the gel beads was approximately $3.2 \mathrm{~mm}(17.9 \mathrm{mg})$. Beads were dried at room temperature in air at a relative humidity $(\mathrm{RH})=11 \%$ (mass after drying, $0.6 \mathrm{mg}$ ). In some cases, a washing step was applied to remove excess (unbound) calcium ions from the alginate beads prior to drying. Gel beads were dispersed in $500 \mathrm{ml}, 1 \mathrm{wt} . \% \mathrm{NaCl}$ and stored for $30 \mathrm{~min}$. The beads were taken out and stored in $500 \mathrm{ml}$ demineralised water for another $30 \mathrm{~min}$. The beads were collected and dried in air.

\section{Preparation of Alginate-CMC Mixed Gel Beads}

Mixed gel beads were prepared by dripping a mixture of sodium alginate and carboxymethyl cellulose (Acatris CMC, grade no. 30) into a 1 wt. $\% \mathrm{CaCl}_{2} \cdot 2 \mathrm{H}_{2} \mathrm{O}$ solution (as described above). The amount of CMC used in the mixture was varied between 0 and 1 wt. $\%$, while the alginate concentration was kept fixed at 1 wt.\%. It was observed that a (small) part of the CMC was lost due to diffusion from the gel bead into the surrounding calcium ion solution. Diffusion losses were minimised by adding $\mathrm{CMC}$ to the calcium ion bath at a concentration between 0 and 1 wt.\% (depending on the CMC concentration in the alginate-CMC mixture). The beads were kept in the solution for $1 \mathrm{~h}$ and then taken out and dried at $11 \%$ RH. The mass of the beads (both wet and dry) was found to depend on the alginate to $\mathrm{CMC}$ ratio. The mass of wet beads (directly after collecting from the solution) was $17.9 \mathrm{mg}(0 \% \mathrm{CMC}), 15.0 \mathrm{mg}(0.1 \%$ CMC), $20.4 \mathrm{mg}(0.3 \% \mathrm{CMC}), 25.3 \mathrm{mg}(0.5 \% \mathrm{CMC})$ and $31.5 \mathrm{mg}(1 \% \mathrm{CMC})$, respectively. The mass of dry beads was $0.6 \mathrm{mg}(0 \% \mathrm{CMC}), 0.5 \mathrm{mg}(0.1 \% \mathrm{CMC})$, $0.6 \mathrm{mg}(0.3 \% \mathrm{CMC}), 0.8 \mathrm{mg}(0.5 \% \mathrm{CMC})$ and $1.1 \mathrm{mg}$ (1\% CMC), respectively.

\section{Preparation of Alginate Gel Fibres}

Calcium alginate fibres were prepared by extruding a sodium alginate solution into a 1 wt. $\% \mathrm{CaCl}_{2} \cdot 2 \mathrm{H}_{2} \mathrm{O}$ solution. The fibres were immediately collected from the solution and air-dried (RH 11\%). During drying, the fibres were stretched to promote alignment of the alginate chains. An annealing step $\left(75^{\circ} \mathrm{C}\right.$ at high humidity for $\left.48 \mathrm{~h}\right)$ was applied after drying to further increase crystallinity of the fibres. 
Preparation of Alginate Gels by Dialysis

A 1-wt.\% sodium alginate solution was poured into a cylindrical bottle capped with a dialysis membrane. The bottle was placed in a 1 -wt. $\% \mathrm{CaCl}_{2} \cdot 2 \mathrm{H}_{2} \mathrm{O}$ solution and $\mathrm{Ca}^{2+}$ ions were allowed to diffuse through the membrane. The diffusion process gave rise to a gradient of $\mathrm{Ca}^{2+}$ ions throughout the sample. After 5 days, the bottle was removed from the calcium ion solution and the alginate gel was cut into approximately $1-\mathrm{mm}$-thin slices. The different slices are characterized by different calcium concentrations, which was clearly noticeable from differences in gel strength. The slices were dried in air at RH $11 \%$.

\section{Rehydration Measurements}

Dried calcium alginate beads were immersed in excess Millipore water (five beads per $100 \mathrm{ml}$ ) or in an aqueous salt solution $\left(\mathrm{NaCl}, \mathrm{KCl}, \mathrm{MgCl}_{2}\right)$ at $20^{\circ} \mathrm{C}$ without stirring. During rehydration, the increase of sample weight was measured as a function of time. Beads were withdrawn from the liquid at regular time intervals and excess water was carefully removed by blotting on a tissue paper before weighing. Rehydration is expressed as

$R(t)=\frac{m(t)-m_{d}}{m_{0}-m_{d}}$,

where $m_{0}$ represents the mass of the bead before drying; $m_{\mathrm{d}}$ represents the mass of the bead after drying and $m(t)$ is the mass of the bead during rehydration. Clearly, $R=1$ corresponds to a situation where the gel beads are hydrated to the same extent as fresh beads before drying; $R>1$ corresponds to a situation where the mass of the rehydrated beads exceeds that of fresh beads. All rehydration measurements were performed in triplicate.

\section{Ca release Measurements}

During rehydration, calcium ions were released from the alginate beads into the surrounding solution. The calcium concentration in solution was monitored using a calciumsensitive electrode (WTW, Nova Atlantis). The solution was slowly stirred to ensure a homogeneous distribution of calcium ions throughout the solution. The amount of calcium released is expressed as a percentage of the total amount of calcium present in the (dried) beads. The latter amount was determined by atomic absorbance to be $0.07 \mathrm{mg}$ calcium per gel bead (for beads prepared from low-G alginate).

\section{X-ray Diffraction}

X-ray diffraction measurements were performed on a Bruker D8 Discovery equipped with a $\mathrm{Cu} k \alpha$ source
$(0.154 \mathrm{~nm})$ and a two-dimensional gas filled multi-wire detector. The two-dimensional scattering patterns were integrated along the azimuthal angle into one-dimensional intensity curves (intensity is displayed as a function of $2 \theta$, where $\theta$ is the scattering angle). All measurements were carried out in transmission geometry.

\section{Kinetic Modelling}

Rehydration was modelled using the following empirical equation:

$R(t)=R_{\mathrm{eq}} \times\left(1-\exp -\left(\frac{t}{\beta}\right)^{\alpha}\right)$.

Equation 2 is also known as the Weibull equation and was previously found to give a good description of rehydration kinetics for a variety of dried foods ${ }^{9-12}$. It has also been used to describe experimental dissolution data and drug release ${ }^{13}$. In Eq. 2, $R(t)$ is the rehydration index as defined in Eq. 1 and $R_{\mathrm{eq}}$ is the rehydration index at equilibrium. $\alpha$ is a shape parameter; its value has a significant effect on the shape of the rehydration curve. For $\alpha<1$, Eq. 2 describes a concave-upward curve whereas for $\alpha>1$ the model predicts a sigmoidal curve with an initial lag phase. The length of the lag phase increases with increasing value of $\alpha . \beta$ is a scale or rate parameter and represents the time needed for $R / R_{\text {eq }}$ to reach a value of $1-e^{-1}$ (i.e. to accomplish approximately $63 \%$ of the rehydration process). A similar expression was used to describe the release of calcium from the gel beads into the surrounding solution:

$C(t)=C_{\mathrm{eq}} \times\left(1-\exp -\left(\frac{t}{\beta}\right)^{\alpha}\right)$,

where $C(t)$ is the fraction calcium released from the beads at time $t$ and $C_{\mathrm{eq}}$ is the fraction calcium released at equilibrium (expressed as a percentage of the total amount of calcium present in dried beads).

\section{Results}

Rehydration of Calcium Alginate Beads in Aqueous Electrolyte Solutions

Calcium alginate beads were rehydrated in different electrolyte solutions $\left(\mathrm{NaCl}, \mathrm{KCl}\right.$ and $\left.\mathrm{MgCl}_{2}\right)$. As an example, Figure 1 shows results for high- and low-G alginate beads in a $10-\mathrm{mM} \mathrm{NaCl}$ solution. The rehydration curve ( $R$ versus time) of high-G alginate beads has a pronounced sigmoidal shape, i.e. the rehydration rate is very low in the initial stage of the process ("lag phase") but 


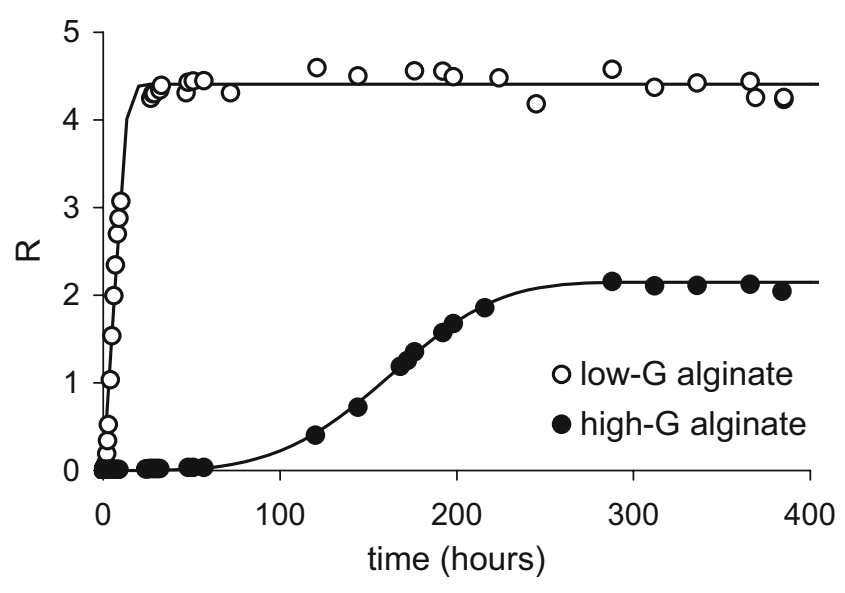

Fig. 1 Rehydration of low- and high-G alginate beads in $10-\mathrm{mM}$ $\mathrm{NaCl}$ solution. Drawn lines are best fits based on Eq. 2 with $\alpha=1.9$, $\beta=8.4 \mathrm{~h}, R_{\mathrm{eq}}=4.41$ (low-G alginate) and $\alpha=3.82, \beta=178 \mathrm{~h}, R_{\mathrm{eq}}=2.15$ (high-G alginate)

considerably increases at a later stage. Equilibrium is reached after approximately $300 \mathrm{~h}$; at this stage, the beads are highly swollen and the mass of the rehydrated particles is larger than the initial mass before drying (i.e. $R_{\text {eq }}>1$ ). Low-G alginate beads are seen to rehydrate considerably faster than beads prepared from high-G alginates. Equilibrium is reached after approximately $20 \mathrm{~h}$ and the particles take up considerably more water than beads prepared from high-G alginate.

Neither low- nor high-G alginate beads were found to rehydrate in pure water (i.e. in the absence of salt). The result suggests that rehydration of calcium alginate beads is dependent on the ionic strength of the rehydration medium. Figure 2 shows the effects of salt concentration $(\mathrm{NaCl}, \mathrm{KCl}$ and $\mathrm{MgCl}_{2}$ ) on the rehydration of low-G alginate beads. For clarity, only the initial part of the rehydration curves is shown. Rehydration rates are seen to increase with increasing salt concentration. At the highest salt concentrations used $(50 \mathrm{mM}$ in case of $\mathrm{NaCl}$ or $\mathrm{KCl}$ and $10 \mathrm{mM}$ in case of $\mathrm{MgCl}_{2}$ ), disintegration of alginate beads was observed after several hours of hydration. Equation 2 was found to give an adequate description of the rehydration curves. Drawn lines in Figure 2 represent fits of Eq. 2 to the experimental data points (note: Eq. 2 was fitted to data points over the entire time range of the experiment, i.e. approximately $60 \mathrm{~h}$ ). Values of the shape parameter $(\alpha)$, scale parameter $(\beta)$ and swelling ratio at equilibrium $\left(R_{\mathrm{eq}}\right)$ are summarised in Table 1.

Release of Calcium from Alginate Beads during Rehydration

During rehydration, calcium ions were released from the alginate beads into the surrounding solution. The release of calcium was found to depend on ionic strength as well as on the type of salt in solution $\left(\mathrm{NaCl}, \mathrm{KCl}\right.$ or $\left.\mathrm{MgCl}_{2}\right)$. As an example, Figure 3 shows results for low-G alginate beads in $50 \mathrm{mM} \mathrm{NaCl}$. It is seen that a large fraction of the calcium initially present in the beads is released during the first approximately $60 \mathrm{~min}$ of rehydration. The effect of salt concentration and ion type on the total amount of calcium released after equilibration, $C_{\text {eq }}$, is summarised in Table 2 .
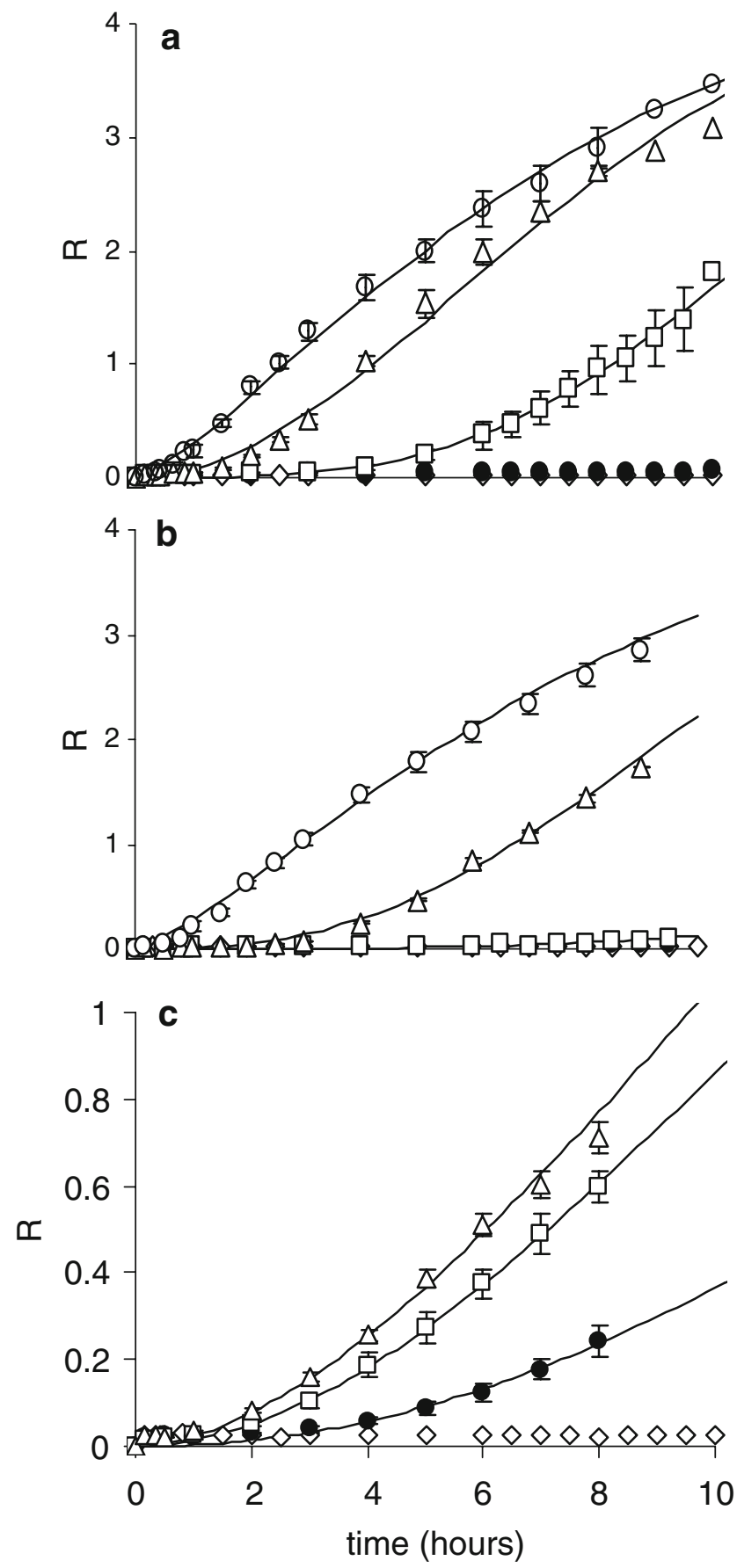

Fig. 2 Rehydration of low-G calcium alginate beads in $(a) \mathrm{NaCl},(b)$ $\mathrm{KCl}$ and $(c) \mathrm{MgCl}_{2}$ solutions at different concentrations: (open circles) $50 \mathrm{mM}$, (open triangles) $10 \mathrm{mM}$, (open squares) $5 \mathrm{mM}$, (full circles) $2 \mathrm{mM}$ and (open diamonds) $0 \mathrm{mM}$. Drawn curves are fits based on Eq. 2; values of $\alpha, \beta$ and $R_{\text {eq }}$ are summarised in Table 1 
Table 1 Rehydration index $\left(R_{\text {eq }}\right)$, shape parameter $(\alpha)$ and rate parameter $(\beta)$ for low- $G$ alginate beads in different salt solutions

\begin{tabular}{lcccc}
\hline Salt $(\mathrm{mM})$ & & $R_{\text {eq }}$ & $\alpha$ & $\beta(\mathrm{h})$ \\
\hline $\mathrm{NaCl}$ & 0 & 0.02 & - & - \\
& 2 & 0.06 & - & - \\
& 5 & 3.87 & 3.3 & 11.9 \\
& 10 & 4.41 & 1.9 & 8.4 \\
& 50 & $(4.6)$ & $(1.5)$ & $(7.7)$ \\
$\mathrm{KCl}$ & 2 & 0.07 & - & - \\
& 5 & 3.30 & 3.3 & 26.7 \\
& 10 & 4.10 & 2.6 & 11.0 \\
& 50 & $(4.45)$ & $(1.3)$ & $(8.4)$ \\
$\mathrm{MgCl}_{2}$ & 2 & 2.1 & 2.1 & 22.0 \\
& 5 & 2.3 & 1.9 & 15.0 \\
& 10 & $(3.0)$ & $(1.8)$ & $(16.0)$ \\
& & & &
\end{tabular}

Values between brackets could not be determined accurately due to disintegration of beads after several hours of rehydration

Note that $C_{\text {eq }}$ increases with increasing salt concentration. The kinetics of calcium release is described adequately by Eq. 3. The drawn line in Figure 3 is a best fit based on Eq. 3 with $\alpha=0.4, \beta=0.09(\mathrm{~h})$ and $C_{\mathrm{eq}}=0.59$. The effect of salt on the parameters $\alpha$ and $\beta$ is summarised in Table 2 . For comparison, Figure 3 also shows a rehydration curve for low- $\mathrm{G}$ alginate beads in $50 \mathrm{mM} \mathrm{NaCl}$. Rehydration is seen to be relatively slow as compared to calcium release: a large fraction of calcium is released before any significant swelling of beads is observed. Similar results were obtained at other salt conditions used (data not shown). The results suggest that rehydration of calcium alginate beads comprises two subsequent steps: release of calcium ions, most likely due to an ion exchange process, followed by swelling of the alginate gel network. The existence of a two-step mechanism might explain the sigmoidal shape of the rehydration curves and the occurrence of a lag phase (which is most clearly seen in the case of high-G alginates). This will be discussed in more detail below.

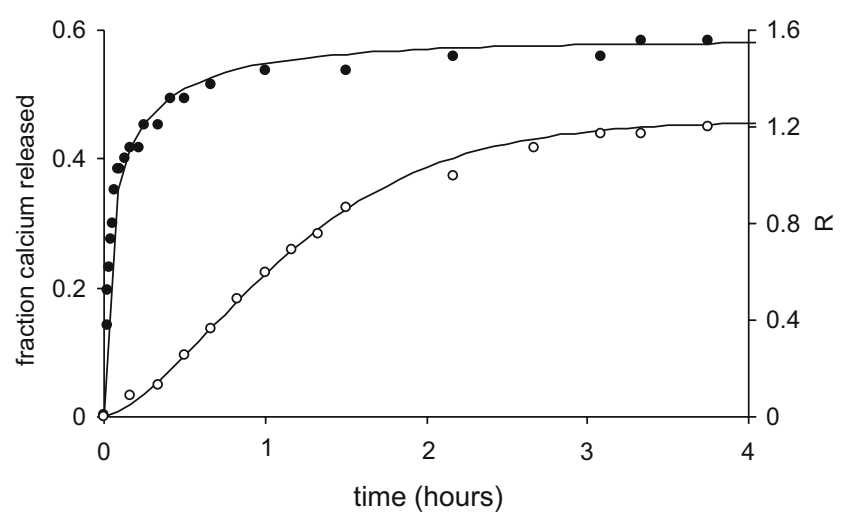

Fig. 3 Release of calcium (full circles) and rehydration (open circles) of low-G alginate beads in $50 \mathrm{mM} \mathrm{NaCl}$. Drawn lines are best fits based on Eqs. 2 and 3
Table 2 Calcium release from low-G alginate beads during rehydration in different salt solutions $\left(C_{\mathrm{eq}}, \alpha\right.$ and $\beta$ were determined by fitting of Eq. 3 to the experimental data)

\begin{tabular}{lrlll}
\hline Salt $(\mathrm{mM})$ & & $C_{\text {eq }}$ & $\alpha$ & $\beta(\mathrm{h})$ \\
\hline $\mathrm{NaCl}$ & 5 & 0.09 & 0.5 & 0.07 \\
& 50 & 0.59 & 0.4 & 0.09 \\
$\mathrm{KCl}$ & 5 & 0.13 & 0.5 & 0.05 \\
& 50 & 0.61 & 0.4 & 0.09 \\
$\mathrm{MgCl}_{2}$ & 5 & 0.21 & 0.5 & 0.08 \\
& 50 & 0.59 & 0.4 & 0.08 \\
\hline
\end{tabular}

\section{Application of a Washing Step}

A washing step was applied to remove excess (unbound) calcium ions from the alginate beads prior to drying. Washing caused significant swelling of the beads but no disintegration was observed. Beads were then air-dried (as before) and subsequently rehydrated in pure water (no salt was added). Rehydration curves of washed and non-washed alginate beads are shown in Figure 4. A washing step is seen to have a significant effect on rehydration: washed beads quickly rehydrate whereas non-washed alginate beads do not absorb any water on the timescale of the experiment. Note that the washed alginate beads start to swell immediately after dispersion in water and that no lag phase is observed. A fit of Eq. 2 to the rehydration curve yielded $\alpha=0.86, \beta=0.72 \mathrm{~h}$ and $R_{\mathrm{eq}}=7.6$.

\section{Rehydration of Alginate-CMC Mixed Gel Beads}

Mixed alginate-CMC gel beads were rehydrated in 5-mM $\mathrm{NaCl}$ solution. Rehydration curves are shown in Figure 5. Beads prepared from a mixture of $1 \mathrm{wt} . \%$ alginate and 0.3 , 0.5 or $1.0 \mathrm{wt} . \% \mathrm{CMC}$ are seen to rehydrate faster than pure alginate beads, whereas beads prepared from a mixture of 1 wt.\% alginate and $0.1 \mathrm{wt} \% \mathrm{CMC}$ rehydrate somewhat slower. In the calculation of the rehydration index $R$, values of $m_{0}$ (mass of fresh beads) and $m_{\mathrm{d}}$ (mass of beads after drying) were used as specified in the "Materials and Methods". Drawn lines in Figure 5 are fits to the experimental data points based on Eq. 2 (values of $R_{\text {eq }}, \alpha$ and $\beta$ could not be determined uni-equally in this case because data points are available for a limited time range only; values were not tabulated for this reason).

\section{X-ray Diffraction}

Figure 6 shows a wide-angle X-ray diffraction pattern of air-dried calcium alginate beads. The pattern shows two broad diffraction peaks centred at $2 \theta=13^{\circ}$ and $2 \theta=23^{\circ}$. The figure also shows a diffraction pattern of washed alginate beads. A broad peak at $2 \theta=23^{\circ}$ is observed, as in the case 


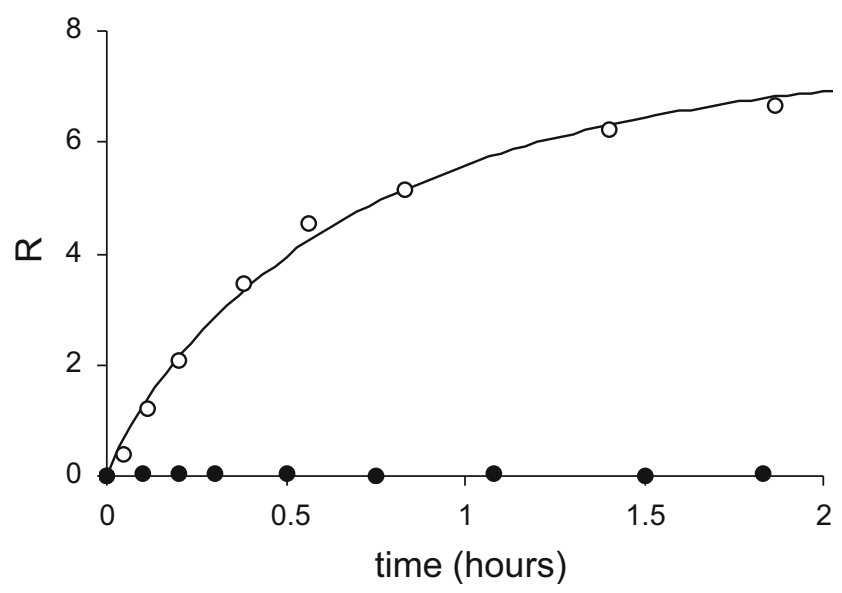

Fig. 4 Rehydration of low-G alginate beads in pure water (no salt present). Open circles: after washing step; full circles: without washing step. Drawn line through open circles is best fit based on Eq. 2 with $\alpha=0.86, \beta=0.72$ (h) and $R_{\text {eq }}=7.6$

of non-washed alginate beads, but a diffraction peak at $2 \theta=$ $13^{\circ}$ is missing. The absence of a diffraction peak at $13^{\circ}$ indicates that the microstructure of washed alginate beads (after air drying) is clearly different from that of non-washed alginate beads. It was speculated that the features that give rise to the diffraction peak at $2 \theta=13^{\circ}$ play an important role in the rehydration behaviour of alginate beads. Therefore, an attempt was made to study the nature of the diffraction peaks in more detail using calcium alginate fibres.

In general, fibre X-ray diffraction studies are the preferred method for establishing the three-dimensional structure of (partially crystalline) polymer materials. Figure $7 \mathrm{a}$ shows a two-dimensional diffraction pattern of calcium alginate fibres. The alginate fibres were annealed at high temperature and humidity for $48 \mathrm{~h}$ (as described in the "Materials and Methods") to enhance the level of crystal-

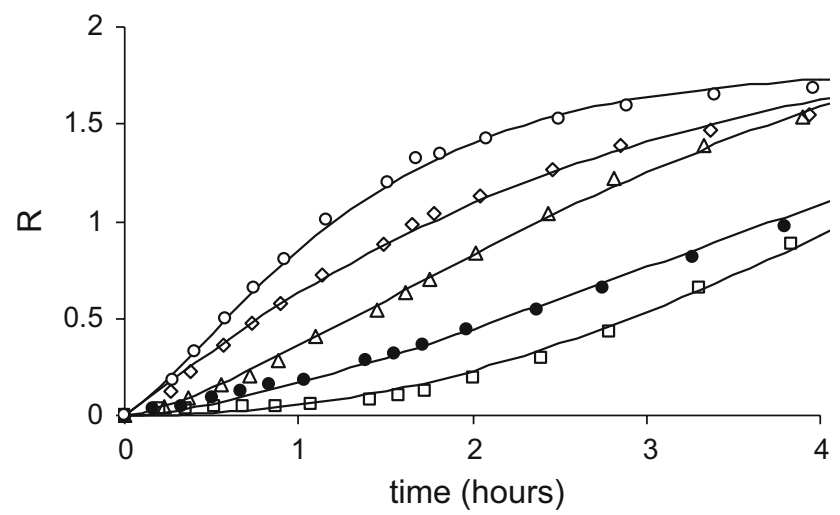

Fig. 5 Rehydration of alginate-CMC mixed gel beads in $5 \mathrm{mM} \mathrm{NaCl}$ solution. Beads were prepared from a mixture of $1 \%$ alginate and (full circles) $0 \% \mathrm{CMC}$, (open squares) $0.1 \% \mathrm{CMC}$, (open triangles) $0.3 \%$ $\mathrm{CMC}$, (open diamonds) $0.5 \% \mathrm{CMC}$ and (open circles) $1 \% \mathrm{CMC}$, respectively. $R$ was calculated using values of $m_{\mathrm{d}}$ and $m_{0}$ given in the "Materials and Methods". Drawn lines are fits based on Eq. 2

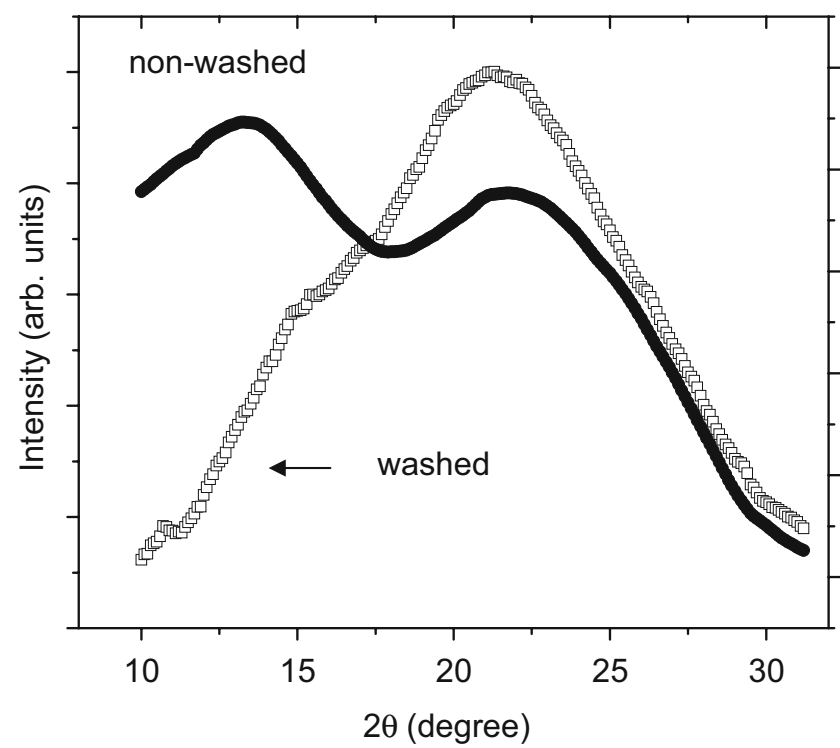

Fig. 6 One-dimensional X-ray diffraction pattern of air-dried calcium alginate beads

linity. The corresponding one-dimensional diffraction curve (obtained by integration of a circular sector along the azimuthal angle) is shown in Figure $7 \mathrm{~b}$. The fibre pattern resembles that of calcium alginate beads (Figure 6): two broad diffraction peaks are observed centred at $2 \theta=13^{\circ}$ and $21.5^{\circ}$, respectively. The arrows in Figure 7 are used to indicate the correlation between the two- and onedimensional patterns: the peak at $2 \theta=13^{\circ}$ results from an area of high intensity in equatorial direction, while the peak at $2 \theta=21.5^{\circ}$ is due to a high intensity in meridian direction. Without any detailed structure interpretation, the result already shows that the scattering peak at $2 \theta=13^{\circ}$ originates from lateral packing of alginate molecules (i.e. perpendicular to the molecular chain direction) and the peak at $2 \theta=21.5^{\circ}$ corresponds to the layer spacing along the molecular chain direction. Following the early work of Atkins et. al. ${ }^{14}$, the peaks at $2 \theta=13^{\circ}$ and $21.5^{\circ}$ are labelled as (110) and (002), respectively (the fibre axis is defined as c-axis). Note that a small shoulder can be observed at $2 \theta=10.6^{\circ}$ in the diffraction pattern in Figure $7 \mathrm{~b}$; the origin
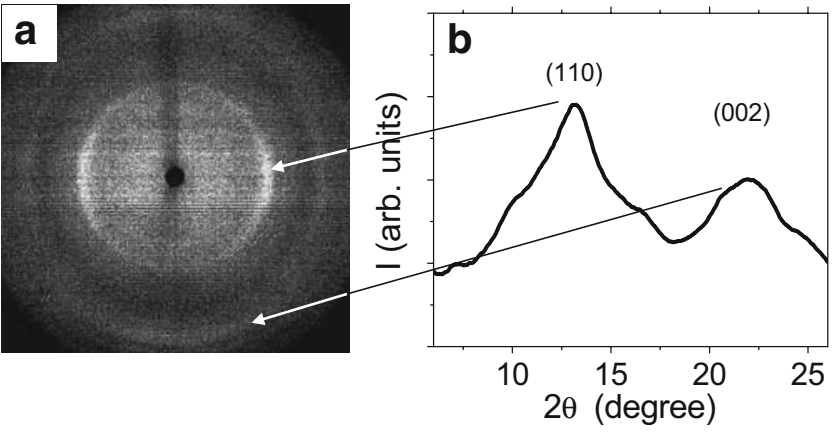

Fig. 7 Two-dimensional (a) and corresponding one-dimensional (b) $\mathrm{X}$-ray scattering pattern of air-dried calcium alginate fibres 
of this shoulder has been studied in detail in our laboratory and is discussed in another paper ${ }^{15}$.

Figure 8 displays X-ray scattering patterns of air-dried alginate samples with different calcium concentrations (the samples were prepared using the dialysis membrane method as explained in the "Materials and Methods"). At the highest calcium concentration, the pattern again shows two broad diffraction peaks centred at $2 \theta=13^{\circ}$ and $23^{\circ}$. At lower calcium concentration, the intensity of the peak at $\sim 13^{\circ}$ (which is associated with the lateral packing of alginate chains) decreases and the peak width increases. The decrease of intensity and the increase of peak width indicate that the number of (lateral) cross-links decreases with decreasing calcium concentration. On the other hand, the intensity of the peak at $23^{\circ}$ is more or less independent of calcium concentration. This observation suggests that this peak does not result from intermolecular cross-linking but from a regular spacing along the molecular chain axis, in line with results from fibre diffraction measurements.

Figure 9 displays $\mathrm{X}$-ray scattering patterns of mixed alginate-CMC gel beads (after air drying). The patterns again show two broad diffraction peaks centred at around $2 \theta=13^{\circ}$ and $23^{\circ}$, respectively. The intensity of the peak at $23^{\circ}$ is independent of the alginate to $\mathrm{CMC}$ ratio (within experimental error). The intensity of the peak at $13^{\circ}$ on the other hand varies with alginate to $\mathrm{CMC}$ ratio. A relatively high peak intensity is observed for beads prepared from a $1 \%$ alginate $-0.1 \%$ CMC mixture; beads prepared from mixtures containing $0.3 \%, 0.5 \%$ or $1.0 \%$ CMC show relatively low peak intensities at $13^{\circ}$. The latter result indicates that lateral packing of alginate chains is reduced in the presence of a sufficiently high amount of CMC. It is speculated that $\mathrm{CMC}$ chains cause steric hindrance and

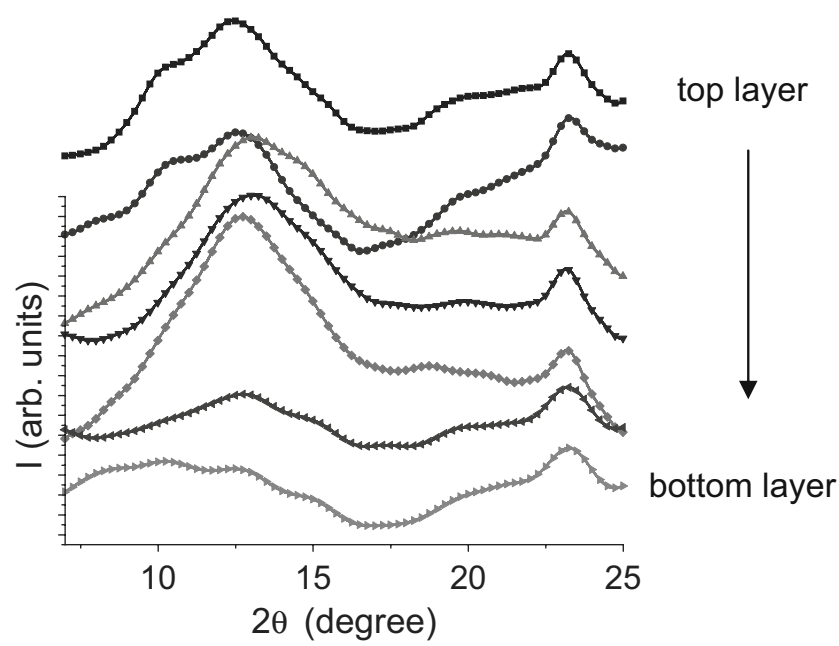

Fig. 8 X-ray diffraction patterns of air-dried alginate samples prepared according to the dialysis membrane method. Calcium concentration in the samples decreases from top (sample closest to dialysis membrane) to bottom. Curves are shifted vertically for clarity of presentation

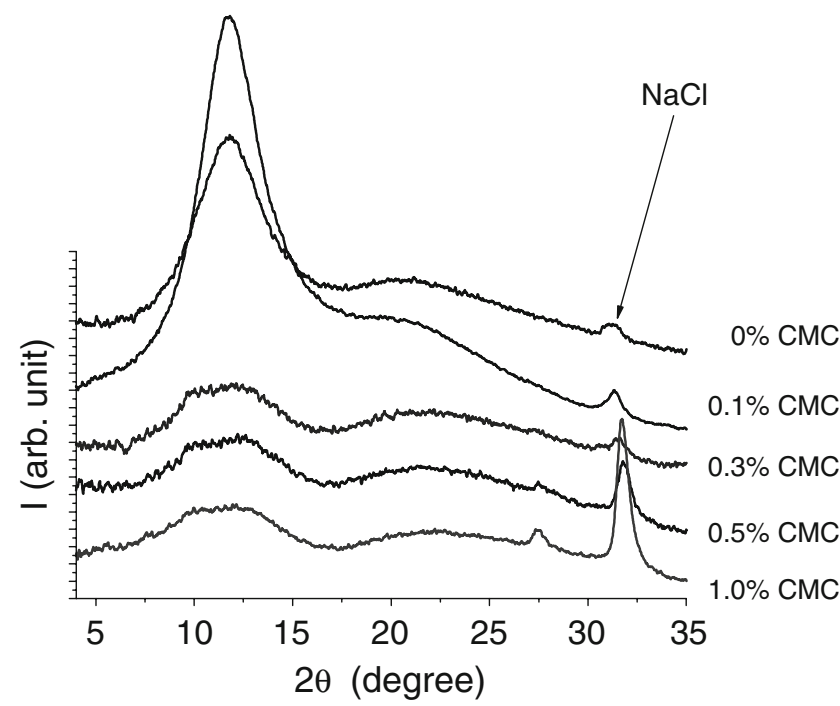

Fig. 9 X-ray diffraction patterns of air-dried alginate-CMC mixed gel beads. Curves are shifted vertically for clarity of presentation

interfere with formation of ordered alginate domains during drying. It is of interest to note that a lower extent of ordering is accompanied by improved rehydration properties of mixed alginate-CMC beads (c.f. Figure 5). This will be discussed in further detail below. At this stage, we cannot explain the high peak intensity in the pattern of beads prepared with a low amount of CMC (0.1 wt.\%). The scattering patterns in Figure 9 further show a sharp peak at $2 \theta=32^{\circ}$. This peak is believed to be associated with the presence of $\mathrm{NaCl}$ crystallites formed during drying of the gel beads.

\section{Discussion}

We propose a simple model to explain the effect of drying on the microstructure and rehydration properties of alginate gel beads. The model is depicted in Figure 10. In freshly prepared gel beads, junction zones are assumed to consist of dimeric units ("egg-box dimers"). Upon drying, dimers are supposed to grow into larger structures, consisting of more than two alginate chains ("egg-box multimers"). The newly formed multimer structures give rise to the scattering peak observed in the X-ray pattern $\left(\right.$ at $2 \theta=13^{\circ}$ ) of air-dried alginate gel beads. Although the exact nature of the multimer structures is not known, we speculate that multimers are formed by lateral association of egg-box dimers. Lateral association of dimers has been shown to occur in alginate gels at high calcium concentration (i.e. high calcium to guluronic acid ratio) $)^{16,17}$. Calcium concentration strongly increases during dehydration of alginate gel beads, especially in the later stage of the drying process. The increase of calcium concentration is assumed to promote association of egg-boxes during drying. In addition, other 


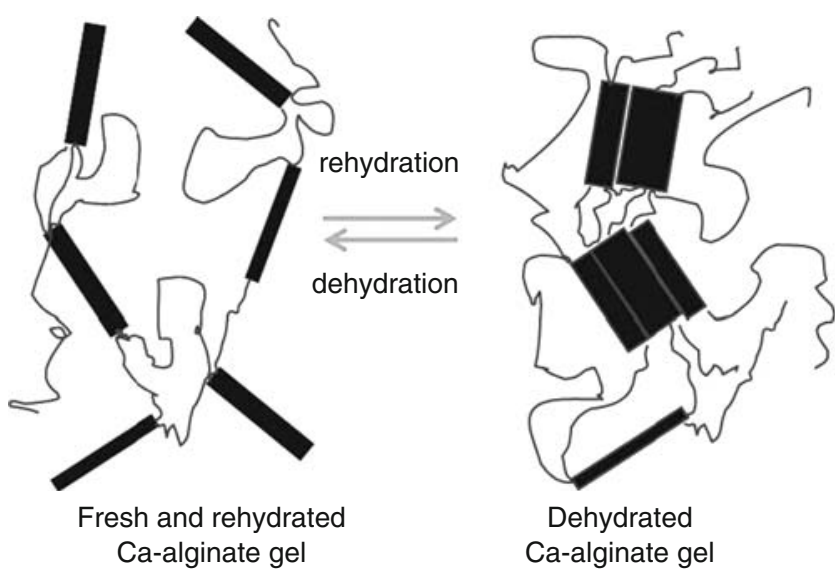

Fig. 10 Formation and breakup of egg-box multimer structures during drying and rehydration in an aqueous salt solution (reprinted from Li et al. ${ }^{15}$ )

processes may contribute to multimer formation. For example, one may speculate that initially (i.e. before drying) only relatively long guluronate segments are involved in junction zone formation. Upon drying, shorter guluronate segments or mixed mannuronic-guluronic blocks may join in and become involved in junction zone formation ${ }^{18}$. It is noted that multimer structures are not formed in the absence of calcium ions (i.e. when calcium ions are removed in a washing step before drying, c.f. Figure 6). This confirms the importance of calcium ions in the formation of multimer structures.

Air-dried alginate beads do not rehydrate to a measurable extent in pure water. Apparently, the junction zones formed during drying are stable in pure water and prevent swelling. Swelling is observed, on the other hand, when airdried beads are dispersed in aqueous salt solutions $\left(\mathrm{Na}^{+}, \mathrm{K}^{+}\right.$ or $\left.\mathrm{Mg}^{2+}\right)$. This suggests that egg-box multimers are disrupted in the presence of (non-cross-linking) salt ions. The stability of dimer-dimer aggregates has been evaluated by Braccini and Perez ${ }^{19}$ using a molecular modelling approach. Their calculations indicate that dimers can associate in many different arrangements, held together by mainly electrostatic $\left(\mathrm{COO}^{-}-\mathrm{Ca}^{2+}-\mathrm{COO}^{-}\right)$interactions. The calculations further indicate that inter-dimer associations are weaker and more tenuous than associations between alginate chains in a single dimer. One could speculate, therefore, that dimer-dimer associations are easily disrupted in the presence of competing salt ions, allowing gel beads to swell in a salt solution. Individual egg-box dimers would remain intact and thus prevent complete dissolution of the gel beads in a salt solution (except at the highest salt concentrations used).

Monovalent cations show different affinities towards alginates. Sodium ions show a stronger binding affinity than potassium and are more effective in competing with calcium ions for binding sites during gel formation ${ }^{20}$. One might speculate therefore that sodium ions are perhaps also more effective in breaking dimer-dimer associations (via an exchange reaction between $\mathrm{Na}^{+}$and $\mathrm{Ca}^{2+}$ ) than potassium ions. This would give rise to faster rehydration kinetics in aqueous $\mathrm{NaCl}$ solutions as compared to $\mathrm{KCl}$ solutions (at equal molar concentration). Rehydration rates appear to be slightly higher in 5- and $10-\mathrm{mM} \mathrm{NaCl}$ solutions, but no significant differences between $\mathrm{NaCl}$ and $\mathrm{KCl}$ solutions were observed at higher $(50 \mathrm{mM})$ salt concentration (Table 1). In addition, one would expect divalent ions to be more effective in disrupting dimer-dimer aggregates than monovalent ions. Alginate beads were found to swell in $2-\mathrm{mM} \mathrm{MgCl}_{2}$ solution, whereas no swelling was observed in 2-mM NaCl or $\mathrm{KCl}$ solution (Figure 2). This suggests that $\mathrm{Mg}^{2+}$ ions are indeed more effective in disrupting dimer-dimer aggregates than $\mathrm{Na}^{+}$or $\mathrm{K}^{+}$ions.

The rehydration kinetics of alginate gel beads are adequately described by the Weibull distribution (Eq. 2). This equation was used previously to describe rehydration of porous food particles and dried plant tissues ${ }^{9-12}$. Rehydration in general is a complex process and may involve different physical mechanisms such as capillary flow, convection or diffusion. The Weibull distribution may be used to differentiate between the different mechanisms as the shape parameter $\alpha$ varies with the mechanism of water uptake ${ }^{12}$. For example, when water uptake is governed by Fickian (or Case I) diffusion, the shape parameter is predicted to have a value between $\alpha=0.67$ and 0.81 , depending on the geometry of the particle (and assuming that particle dimensions remain constant during rehydration). Higher values of $\alpha(1.21-1.60)$ are predicted when water uptake is dominated by Case II (non-Fickian) diffusion. Case II diffusion is usually observed in glassy polymer systems when water uptake is dominated by slow plasticization or relaxation phenomena. The $\alpha$ values deduced from the rehydration curves of alginate gel beads are significantly higher $(1.5<\alpha<3.3$; see Table 1$)$ than those predicted for Case I or Case II diffusion, suggesting that other mechanisms are involved in the rehydration of alginate beads. This is in line with the model proposed above, which assumes that rehydration involves breakup of junction zones under influence of salt ions before any significant swelling can take place. Note that $\alpha$ is significantly lower for alginate beads that were washed before drying: $\alpha=0.86$ (Figure 4). This is more close to the value of $\alpha$ anticipated for type I diffusion.

Mixed alginate-CMC gel beads were found to have better rehydration properties than pure alginate beads (except at the lowest CMC concentration used; Figure 5). It is speculated that CMC reduces aggregation of alginateegg-boxes and interferes with formation of multimer structures during drying (possibly via steric hindrance). The latter effect was confirmed by X-ray measurements 
(Figure 9) which show a decrease of the peak intensity at $2 \theta=13^{\circ}$ (associated with multimer formation) in the presence of CMC. Other effects (e.g. osmotic effects or increased pore sizes) may also contribute to better rehydration properties of mixed gel beads. No attempts were made yet to characterise the microstructure of dried alginateCMC gel beads in more detail. At this stage, it is not clear whether $\mathrm{CMC}$ and alginate chains remain intimately mixed (as in the wet state) or whether phase segregation occurs upon drying. Phase segregation upon drying has been reported previously for polymer mixtures ${ }^{21}$ and is a topic of current investigation in our laboratory.

Calcium alginate gel beads show some resemblance to other food biopolymer systems during drying and rehydration. In a recent study, the rehydration properties of bacterial cellulose gels were examined ${ }^{5}$. For these systems, air drying resulted in extensive and irreversible aggregation of cellulose fibres. The formation of aggregates prevented swelling of the cellulose network upon rehydration. Salt ions did not affect swelling behaviour, but various lowmolecular-weight sugars (added to the gel matrix prior to drying) were found to improve the rehydration properties. Scanning electron micrographs confirmed that aggregation of cellulose fibres is strongly reduced in the presence of low-molecular-weight sugars (in particular sucrose and raffinose). It is speculated that low-molecular-weight sugars interfere with the aggregation process, just as CMC interferes with aggregation of alginate chains.

\section{Summary}

Rehydration of air-dried calcium alginate beads was found to depend on the type of alginate used (low-G versus highG) and the salt concentration in the rehydration medium. Rehydration was improved when excess calcium ions were removed from the alginate beads in a washing step prior to drying. Mixed alginate-CMC beads were found to rehydrate faster than pure alginate beads. The rehydration curves, including the occurrence of a lag phase, were described adequately by the empirical Weibull equation. The poor rehydration properties of calcium alginate beads in pure water (i.e. in the absence of salt) are attributed to egg-box multimer structures formed during drying. The existence of such structures was deduced from X-ray scattering patterns. Multimer structures break up in the presence of salt ions, probably via an ion exchange reaction, which explains the improved rehydration behaviour in aqueous salt solutions.

Acknowledgement Nadége Perret and Stephanie Dechaux are acknowledged for their assistance in the rehydration experiments. Rob Groot is acknowledged for valuable discussions.

\section{References}

1. Q. Wang, P.R. Ellis, S.B. Ross-Murhpy, Carbohydr. Polym. 49, 131 (2002)

2. Q. Wang, P.R. Ellis, S.B. Ross-Murhpy, Carbohydr. Polym. 53, 75 (2003)

3. Q. Wang, P.R. Ellis, S.B. Ross-Murhpy, Carbohydr. Polym. 64, 239 (2006)

4. C.K. Larsen, O. Gåserød, O. Smidsrød, Carbohydr. Polym. 51, $125(2003)$

5. R. Vreeker, I. Appelqvist, A. Hol, M. Kirkland, A. Ledeboer, L. Li, L. Lundin, S. Schumm, Effect of Co-solutes on Drying and Rehydration of Biopolymer Networks in Gums and Stabilisers for the Food Industry 13, ed. by P.A. Williams, G.O. Phillips (RSC Publishing, Cambridge, 2006)

6. K.I. Draget, O. Smidsrød, G. Skjåk-Bræk, Alginates From Algae in Polysaccharides and Polyamides in the Food Industry. Properties, Production and Patents, ed. by A. Steinbüchel, S.K. Rhee (Wiley-VCH Verlag GmbH \& Co. KGaA, Weinheim, 2005)

7. G.T. Grant, E.R. Morris, D.A. Rees, P.J. Smith, D. Thom, FEBS Lett. 32, 195 (1973)

8. W. Weibull, J. Appl. Mech. 18, 293 (1951)

9. M.F. Machado, F.A.R. Oliveira, L. Cunha, Int. J. Food Sci. Technol. 34, 47 (1999)

10. L.M. Cunha, F.A.R. Oliveira, A.P. Aboim, J.M. Frías, A. Pinheiro-Torres, Int. J. Food Sci. Technol. 36, 253 (2001)

11. G. Sacchetti, P. Pittia, M. Biserni, G.G. Pinnavaia, M.D. Rosa, Int. J. Food Sci. Technol. 38, 135 (2003)

12. A. Marabi, S. Livings, M. Jacobson, I.S. Saguy, Eur. Food Res. Technol. 217, 311 (2003)

13. K. Kosmidis, P. Argyrakis, P. Macheras, Pharm. Res. 20, 988 (2003)

14. E.D.T. Atkins, I.A. Nieduszynski, W. Mackie, K.D. Parker, E.E. Smolko, Biopolymers 12, 1879 (1973)

15. L. Li, Y. Fang, R. Vreeker, I. Appelqvist, E. Mendes, Biomacromolecules 8, 464 (2007)

16. B.T. Stokke, K.I. Draget, O. Smidsrød, Y. Yuguchi, H. Urakawa, K. Kajiwara, Macromolecules 33, 1853 (2000)

17. Y. Fang, S. Al-Assaf, G.O. Phillips, K. Nishinari, T. Funami, P.A. Williams, L. Li, J. Phys. Chem. B 111, 2456 (2007)

18. I. Donati, S. Holtan, Y.A. Morch, M. Borgogna, M. Dentini, G. Skjak-Braek, Biomacromolecules 6, 1031 (2005)

19. I. Braccini, S. Pérez, Biomacromolecules 2, 1089 (2001)

20. K.I. Draget, K. Steinsvåg, E. Onsøyen, O. Smidsrød, Carbohydr. Polym. 35, 1 (1998)

21. I. Hopkinson, M. Myatt, Macromolecules 35, 5153 (2002) 